\title{
In Patients with Coronary Artery Disease and Type 2 Diabetes, SIRT1 Expression in Circulating Mononuclear Cells Is Associated with Levels of Inflammatory Cytokines but Not with Coronary Lesions
}

\author{
Yuanmin $\mathrm{Li}^{1}{ }^{1}$ Jing $\mathrm{Ni}^{2}$ Rong Guo, ${ }^{2}$ and Weiming $\mathrm{Li}^{2}$ \\ ${ }^{1}$ Department of Cardiovascular Surgery, Shanghai Tenth People's Hospital, Tongji University School of Medicine, \\ Shanghai 200072, China \\ ${ }^{2}$ Department of Cardiology, Shanghai Tenth People's Hospital, Tongji University School of Medicine, Shanghai 200072, China \\ Correspondence should be addressed to Weiming Li; lwmsoncentury@126.com
}

Received 6 December 2015; Revised 29 February 2016; Accepted 13 March 2016

Academic Editor: Ramazan Akdemir

Copyright (C) 2016 Yuanmin Li et al. This is an open access article distributed under the Creative Commons Attribution License, which permits unrestricted use, distribution, and reproduction in any medium, provided the original work is properly cited.

\begin{abstract}
While SIRT1 is significantly associated with atherosclerosis and diabetic complications, its relevance to coronary lesions in patients with coronary artery disease and type 2 diabetes has not been specifically investigated. Thus, we assessed SIRT1 expression in peripheral blood mononuclear cells in these patients. We found that SIRT1 expression did not significantly correlate with syntax scores from coronary angiography $(p>0.05)$. Notably, plasma levels of the inflammatory cytokines tumor necrosis factor- $\alpha$, monocyte chemoattractant protein-1, and high-sensitivity C-reactive protein were markedly higher in diabetic patients $(p<0.05)$. In addition, SIRT1 expression was negatively correlated with levels of these cytokines, as well as that of interleukin-6 $(p<0.05)$. In summary, the data indicate that SIRT1 expression in peripheral blood mononuclear cells is significantly correlated with inflammatory cytokines levels in patients with coronary artery disease and type 2 diabetes but not with the severity of coronary lesions.
\end{abstract}

\section{Introduction}

Type 2 diabetes mellitus (T2DM) and coronary artery disease (CAD) are major public health issues in industrialized countries $[1,2]$. T2DM is a major independent risk factor for CAD and accelerates the development of atherosclerosis via various mechanisms [3]. Consequently, cardiovascular complications are a major cause of mortality and morbidity in diabetic patients [4]. Indeed, patients with both diabetes and CAD are challenging to manage and comprise a growing segment of the population [5]. Therefore, therapeutic strategies for these patients are urgently needed $[6,7]$.

Sirtuin 1 (SIRT1), a nicotinamide adenine dinucleotidedependent histone deacetylase that regulates glucose and lipid metabolism [8-10], is closely associated with extended lifespan due to calorie restriction [11]. Stein and colleagues [12] also found that SIRT1 prevents atherogenesis by inhibiting the formation of macrophage foam cells. Accordingly,
SIRT1 expression has been reported to be significantly lower in patients with acute coronary syndrome or stable CAD $[13,14]$. Finally, evidence has also accumulated to suggest that SIRT1 activation could be a therapeutic strategy to reverse atherosclerosis [15-18]. However, SIRT1 expression and its relationship with coronary lesions in patients with both CAD and T2DM have not been investigated. In this study, we tested the hypothesis that mRNA expression in peripheral blood mononuclear cells correlates with the severity of coronary lesions in diabetic patients.

\section{Materials and Methods}

2.1. Study Population. We screened patients with CAD who were admitted to our department between July 2013 and July 2014. In the end, we enrolled 285 consecutive patients with or without T2DM. Diagnosis of T2DM was established on the 
criteria from guideline [19]. These patients were 20-70 years old, had typically ischemic angina, and had undergone coronary angiography. Patients with a history of ACS $<6$ months prior to enrollment were excluded, along with those who had connective tissue, autoimmune, malignant, or infectious disease within the previous month. Patients with anemia (hemoglobin $<90 \mathrm{~g} / \mathrm{L}$ ) and severe hepatic or renal failure or who had major operations or trauma within the previous 3 months were also excluded. Patients were treated according to routine clinical protocols and guidelines. Standard 12lead electrocardiograms, cardiac biomarker data, plasma inflammatory biomarkers, and coronary angiographic results were collected, along with patient demographics and risk factors such as age, gender, hypertension, smoking status, and hyperlipidemia. Fifty healthy individuals were enrolled as controls. This study was approved by the local institutional ethics committee, and written informed consent was obtained.

2.2. Isolation of Peripheral Blood Monocytes. Blood was suspended over a layer of Ficoll reagent and centrifuged for $20 \mathrm{~min}$ at $1800 \times \mathrm{g}$ and room temperature to separate peripheral blood mononuclear cells. These cells were then transferred to a fresh tube and washed twice with PBS. Subsequently, monocytes were isolated by magnetic-activated cell sorting (MACS), using magnetic beads coated with CD14. The purity of isolated monocytes was tested by flow cytometry as described [14], using cells stained with fluoresceinlabeled CD14 antibody.

2.3. RNA Extraction and Quantitative Real-Time PCR. Total RNA was extracted from purified peripheral blood monocytes resuspended in Trizol reagent (Invitrogen, USA). RNA concentration, purity, and quality were assessed by optical density at $260 \mathrm{~nm}$ and $280 \mathrm{~nm}$ and by visualization of $18 \mathrm{~S}$ and $28 \mathrm{~S}$ rRNA. Samples $(1 \mu \mathrm{g})$ were reverse transcribed using oligo(Dt) ${ }_{15}$ and M-MLV Reverse Transcriptase (Promega, USA) according to the manufacturer's instructions. The resulting cDNA $(2 \mu \mathrm{L})$ was amplified by semiquantitative PCR in $25 \mu \mathrm{L}$ reactions containing $12.5 \mu \mathrm{L} 2 \mathrm{x}$ PCR-Mix, $1 \mu \mathrm{L}$ each of forward and reverse primers $(10 \mu \mathrm{mol} / \mathrm{L})$, and sterile water. Primers were designed in Primer 5.0 to span exons to minimize amplification of genomic DNA, if present. SIRT1 primers had sense sequence $5^{\prime}$-CGG ATT TGA AGA ATG TTG GTT C-3' and antisense sequence $5^{\prime}$-GGA-AAA-TGTAAC-GAT-TTG-GTG-G-3'. GAPDH was amplified with sense primer $5^{\prime}$-ACG GAT TTG GTC GTA TTG GG-3' and antisense primer $5^{\prime}$-TGA TTT TGG AGG GAT CTC GC$3^{\prime}$. Genes were amplified by initial denaturation at $94^{\circ} \mathrm{C}$ for $3 \mathrm{~min}$, followed by cycles of denaturation at $94^{\circ} \mathrm{C}$ for $30 \mathrm{~s}$, annealing for $30 \mathrm{~s}$, and extension at $72^{\circ} \mathrm{C}$ for $50 \mathrm{~s}$, and then by final extension at $72^{\circ} \mathrm{C}$ for $5 \mathrm{~min}$. Annealing temperature and number of cycles were optimized for each gene. In particular, the number of cycles was set in preliminary trials to be within the linear range to ensure accuracy of semiquantitative analysis. PCR products $(5 \mu \mathrm{L})$ were then separated on $1.5 \%$ agarose gel and quantified by densitometry using Scion Image. Expression was normalized to GAPDH.
2.4. Coronary Angiography and Syntax Scoring. High-risk patients with CAD, as well as other patients suitable for the procedure according to the latest guidelines, underwent coronary angiography within $72 \mathrm{~h}$ of admission to confirm ischemic angina. The locations of coronary lesions, the number of stenosed arteries, and the degree of stenosis were recorded. Syntax scores were calculated from the angiograms using a validated web-based syntax scoring system [20, 21]. This procedure was done by two experienced cardiologists blinded to clinical data.

2.5. Assay of Inflammatory Cytokines. Blood samples were drawn upon admission into vacuum containers containing ethylenediaminetetraacetic acid and stored at $-80^{\circ} \mathrm{C}$ until analysis. Plasma levels of the inflammatory cytokines interleukin-6 (IL-6), tumor necrosis factor- $\alpha$ (TNF- $\alpha$ ), monocyte chemoattractant protein-1 (MCP-1), and highsensitivity C-reactive protein (hs-CRP) were measured using enzyme-linked immunosorbent assay kits (R\&D Systems, MN, USA). Measurements were collected in duplicate and averaged.

2.6. Statistical Analysis. Values are reported as mean \pm standard deviation. Student's $t$-test or Wilcoxon matched-pairs, signed-ranks test was used to determine differences between groups Gaussian and non-Gaussian data, respectively. $\chi^{2}$ test or Fisher's exact test was used to compare dichotomous data. $p$ values $<0.05$ were considered statistically significant. Data were analyzed in SPSS 16.0 (SPSS, Chicago, IL, USA).

\section{Results}

3.1. Clinical Characteristics. Enrolled patients had mean age of 58.3 years, and $62.4 \%$ were male. Details of patient characteristics are listed in Table 1. Patients with or without T2DM were not significantly different in terms of age, gender distribution, history of hypertension and hyperlipidemia, smoking status, CG-GFR, DBP, and ongoing drug therapy, which included antiplatelet drugs, beta-blockers, ACEI/ARB, statins, diuretics, and CCB. However, there were significant differences in terms of BMI, SBP, heart rate, LVEF, blood glucose, glycosylated hemoglobin, and insulin treatment.

3.2. SIRT1 Expression. The relative expression of SIRT1 in healthy individuals and CAD patients with or without T2DM was $1.85 \pm 0.04,1.64 \pm 0.03$, and $1.50 \pm 0.05$, respectively. Expression was significantly lower in patients with CAD and diabetes (Figure 1; $p<0.01$ ). Additionally, normalized expression at the 25th percentile was 1.2 and at the 75 th percentile 1.8 (Figure 1).

3.3. Syntax Scores. Syntax scores in all patients and in patients with or without T2DM were $29.4 \pm 13.4$, $33.1 \pm 1.4$, and $27.9 \pm 1.3$, respectively. There is no significant difference between diabetic and nondiabetic patients $(p=0.089$, Figure 2). SIRT1 expression did not significantly correlate with syntax scores in CAD patients with or without T2DM (all $p>0.05)$. 
TABLE 1: Demographic and baseline clinical characteristics.

\begin{tabular}{|c|c|c|c|}
\hline & Healthy controls & Patients with CAD & Patients with CAD and T2DM \\
\hline$n$ & 50 & 207 & 78 \\
\hline Age (y) & $37.4 \pm 1.4$ & $58.3 \pm 0.6$ & $58.4 \pm 1.1$ \\
\hline Gender (male, \%) & $31(62 \%)$ & $124(59.9 \%)$ & $54(69.2 \%)$ \\
\hline Hypertension, $n(\%)$ & $0(0)$ & $114(55.1 \%)$ & $44(56.4 \%)$ \\
\hline Hyperlipidemia, $n(\%)$ & $0(0)$ & $120(58.0 \%)$ & $58(66.7 \%)$ \\
\hline Smoking, $n(\%)$ & $0(0)$ & $122(58.9 \%)$ & $47(60.2 \%)$ \\
\hline BMI $\left(\mathrm{kg} / \mathrm{m}^{2}\right)$ & $24.0 \pm 0.4^{*}$ & $24.1 \pm 0.2^{\#}$ & $25.2 \pm 0.3$ \\
\hline CG-GFR $(\mathrm{mL} / \mathrm{min})$ & $92.1 \pm 0.9^{*}$ & $88.1 \pm 0.7$ & $85.4 \pm 1.3$ \\
\hline $\mathrm{SBP}(\mathrm{mmHg})$ & $128.4 \pm 1.8^{*}$ & $131.3 \pm 1.1^{*}$ & $136.2 \pm 2.3$ \\
\hline $\mathrm{DBP}(\mathrm{mmHg})$ & $73.5 \pm 1.3$ & $74.6 \pm 0.7$ & $74.8 \pm 1.2$ \\
\hline Heart rate (beats/min) & $73.6 \pm 1.3^{*}$ & $74.4 \pm 0.8^{*}$ & $77.5 \pm 1.4$ \\
\hline $\operatorname{LVEF}(\%)$ & $63.2 \pm 0.9^{\#}$ & $61.8 \pm 0.5^{\#}$ & $58.8 \pm 0.9$ \\
\hline Fasting blood glucose (mmol/L) & $5.3 \pm 0.3^{\#}$ & $5.5 \pm 0.4^{\#}$ & $7.2 \pm 0.3$ \\
\hline $\operatorname{HbAlC}(\%)$ & $5.2 \pm 0.3^{*}$ & $5.4 \pm 0.2^{*}$ & $6.9 \pm 0.4$ \\
\hline Duration of diabetes (years) & 0 & 0 & $3.4 \pm 1.1$ \\
\hline \multicolumn{4}{|l|}{ Drug therapy } \\
\hline Antiplatelet drugs, $n(\%)$ & $0(0)$ & $133(64.3 \%)$ & $51(65.4 \%)$ \\
\hline Beta-blocker, $n(\%)$ & $0(0)$ & $78(37.7 \%)$ & $30(38.5 \%)$ \\
\hline $\mathrm{ACEI} / \mathrm{ARB}, n(\%)$ & $0(0)$ & $94(45.4 \%)$ & $36(46.2 \%)$ \\
\hline Statins, $n(\%)$ & $0(0)$ & $127(61.4 \%)$ & $42(53.8 \%)$ \\
\hline Diuretics, $n(\%)$ & $0(0)$ & $26(12.6 \%)$ & $9(11.5 \%)$ \\
\hline $\mathrm{CCB}, n(\%)$ & $0(0)$ & $103(49.8 \%)$ & $38(48.7 \%)$ \\
\hline Insulin, $n(\%)$ & $0(0)$ & $0(0)^{\#}$ & $17(21.8 \%)$ \\
\hline
\end{tabular}

BMI: body mass index; CG-GFR: Cockcroft-Gault glomerular filtration rate; SBP: systolic blood pressure; DBP: diastolic blood pressure; LVEF: left ventricular ejection fraction; HbAlC: glycosylated hemoglobin; IL-2: interleukin-2; hs-CRP: high-sensitivity C-reactive protein; TNF- $\alpha$ : tumor necrosis factor- $\alpha$; MCP-1: monocyte chemotactic protein-1; ACEI: angiotensin converting enzyme inhibitor; ARB: angiotensin II receptor blocker; CCB: calcium channel blocker. ${ }^{*} p<$ 0.05 versus CAD patients with diabetes; ${ }^{*} p<0.01$ versus CAD patients with diabetes.

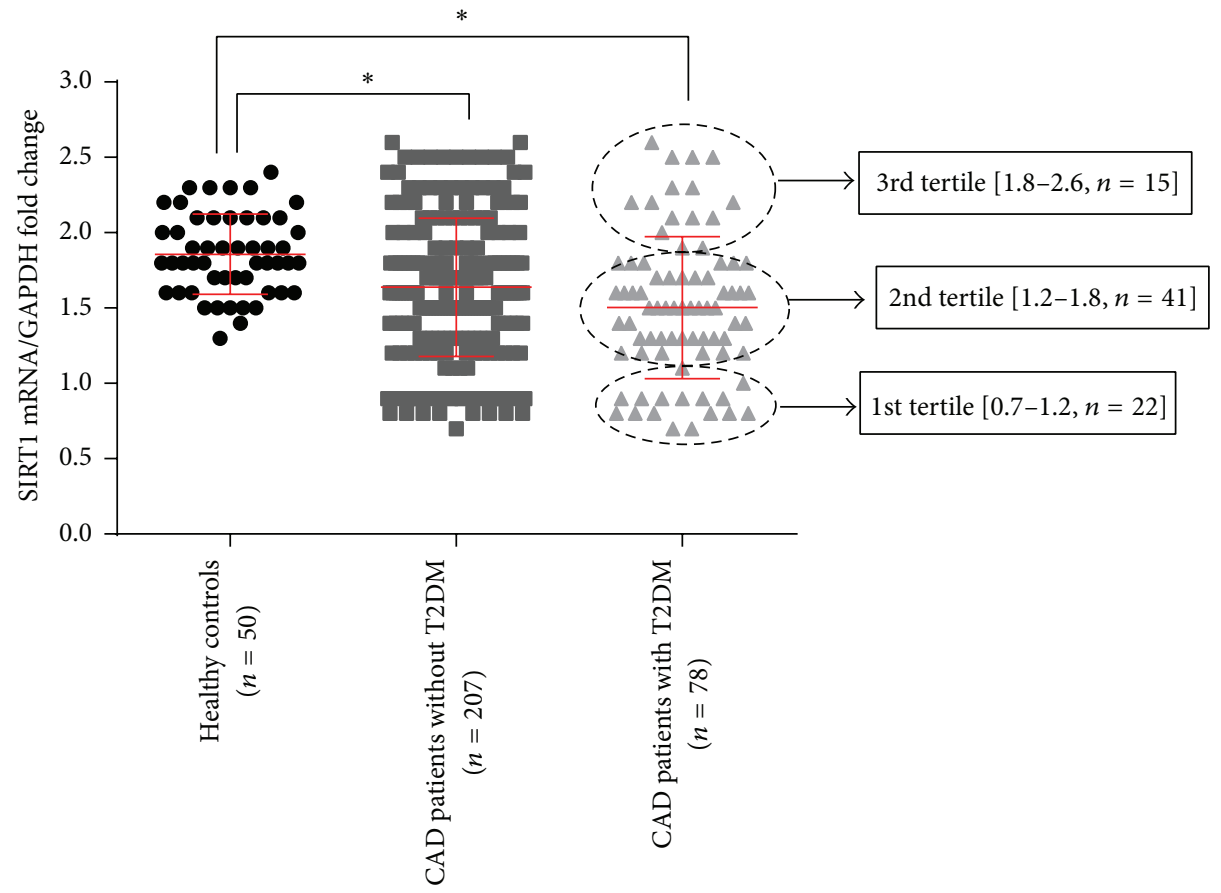

FIGURE 1: mRNA expression of SIRT1. There is no significant difference between patients with or without T2DM. In patients with T2DM, data are divided into tertiles: 1 st tertile $<1.2$, 2nd tertile $500<1.8$, and 3rd tertile $\leq 2.6$. ${ }^{*} p<0.05$. 


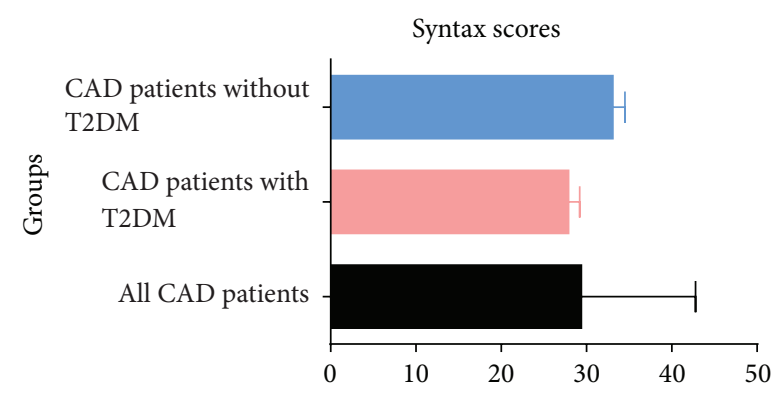

FIGURE 2: Syntax scores in three groups.

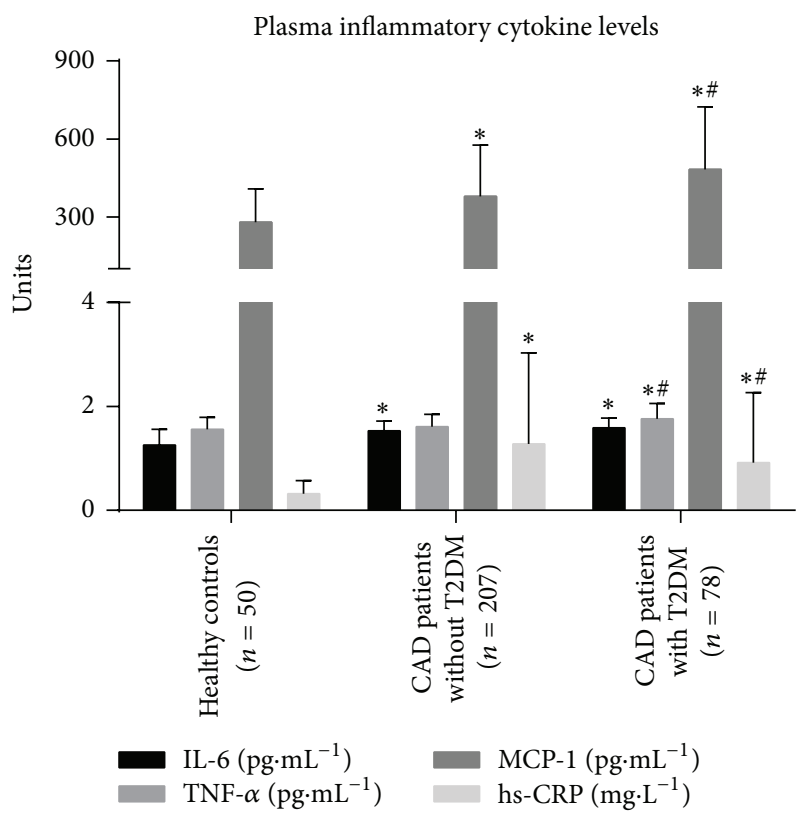

FIGURE 3: Levels of inflammatory cytokines in the plasma. TNF$\alpha, \mathrm{MCP}-1$, and hs-CRP levels are significantly higher in diabetic patients than in nondiabetic patients. ${ }^{*} p<0.05$ versus healthy subjects; ${ }^{*} p<0.05$ versus nondiabetic patients.

3.4. Inflammatory Cytokines and SIRT1 Expression. Circulating levels of IL-6 were $1.26 \pm 0.30,1.59 \pm 0.19$, and $1.53 \pm 0.19 \mathrm{pg} / \mathrm{mL}$ in healthy individuals and in CAD patients with or without T2DM, respectively. The difference between patients with $\mathrm{CAD}$ and healthy controls was significant $(p<0.001)$, but there was no significant difference between diabetic and nondiabetic patients $(p=0.057$, Figure 3$)$. On the other hand, plasma TNF- $\alpha$ in diabetic patients was significantly higher $(p<0.001)$ at $1.76 \pm 0.30 \mathrm{pg} / \mathrm{mL}$ than in nondiabetic patients and healthy controls, who had comparable $(p=0.172)$ TNF- $\alpha$ levels of $1.56 \pm 0.23$ and 1.61 $\pm 0.24 \mathrm{pg} / \mathrm{mL}$, respectively (Figure 3 ). Additionally, plasma hs-CRP was significantly higher in nondiabetic patients than in control subjects and diabetic patients $(p<0.05$ and $p<$ 0.001 , Figure 3 ). Hs-CRP was $0.32 \pm 0.25,1.28 \pm 1.75$, and 0.92 $\pm 1.35 \mathrm{mg} / \mathrm{L}$ in healthy, nondiabetic, and diabetic patients, respectively. Finally, plasma MCP-1 was $279.9 \pm 127.9,378.2$ \pm 198.8 , and $483.4 \pm 240.4 \mathrm{pg} / \mathrm{mL}$ in healthy individuals and nondiabetic and diabetic patients, respectively. The difference between diabetic and nondiabetic patients was significant $(p<0.001$, Figure 3).

The level of TNF- $\alpha$ was found to significantly relate to peripheral SIRT1 expression in monocytes in patients without T2DM $\left(r^{2}=0.026 ; p=0.0193\right)$. There were no significant correlations between SIRT1 expression and other plasma levels (IL-6, hs-CRP, and MCP-1) in this study ( $p=0.0524$, $p=0.3353$, and $p=0.8814)$. However, SIRT1 expression was significantly correlated with levels of inflammatory cytokines in patients with T2DM $(p<0.05$, Figures $4(a)-4(d))$.

\section{Discussion}

Macrovascular complications are common in patients with T2DM who then present accelerated atherosclerotic changes in vessels [22]. Growing evidence suggests that SIRT1 can be regarded as a new therapeutic target to prevent or reverse such complications [23-25]. However, the exact relationship between SIRT1 expression and coronary arterial stenosis is poorly understood. Our data suggest that SIRT1 expression in peripheral blood mononuclear cells is not associated with the severity of coronary lesions in patients with T2DM but is associated with levels of inflammatory cytokines in the plasma. This finding may partly explain outcomes in patients with both CAD and T2DM.

Crujeiras and colleagues [26] assessed SIRT1 expression in peripheral blood mononuclear cells to investigate the effect of the sirtuin pathway on obesity therapy. de Kreutzenberg et al. [27] reported that insulin resistance and metabolic syndrome were associated with low SIRT1 expression in the same cells and that SIRT1 expression was negatively correlated with subclinical atherosclerosis. Song et al. [28] provided some evidence that SIRT1 might have a role in the pathogenesis of T2DM, and its expression in granulocytes and monocytes might indirectly reflect metabolic status in diabetic patients. Many studies have also demonstrated that SIRT1 might suppress inflammation and thus be used to manage inflammatory disorders [29-31]. In accordance with these results, our data link SIRT1 to inflammatory biomarkers in patients with CAD and T2DM.

Sirtuins (SIRT1-SIRT7) are a family of nicotinamide adenine dinucleotide-dependent enzymes. SIRT1, an important member of this family, is involved in a wide range of physiological and pathological processes [32]. For example, SIRT1 promotes reverse cholesterol transport in macrophages by regulating the oxidized low-density lipoprotein receptor1 (LOX-1) and the liver X-receptor (LXR) and by inhibiting formation of foam cells $[12,15]$. On the other hand, SIRT1 overexpression or activation suppresses expression of adhesion molecules in endothelial cells through the NF$\mathrm{Kb}$ pathway and thereby improves endothelial function [16]. Gorenne et al. [33] also found that SIRT1 expression in vascular smooth muscle cells protects against DNA damage, medial degeneration, and atherosclerosis. Indeed, SIRT1 activity may be involved in the development of atherosclerosis and is thus a promising target for novel drugs against CVD and related diseases [34].

Furthermore, SIRT1 has been implicated in glucose homeostasis and lipid metabolism in adipose tissue, liver, 


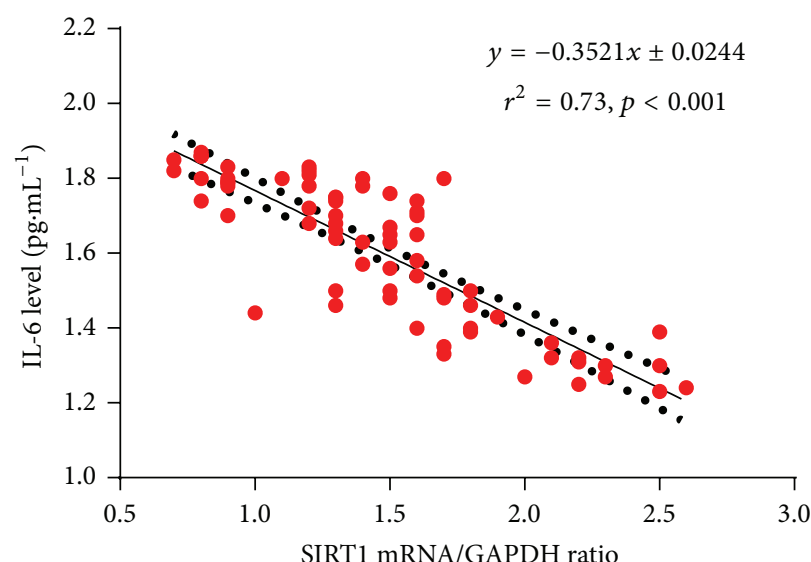

(a)

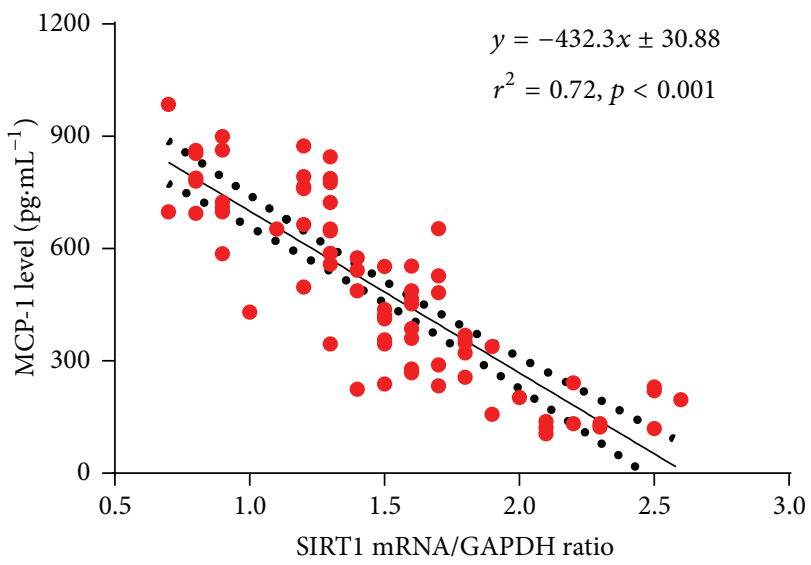

(c)

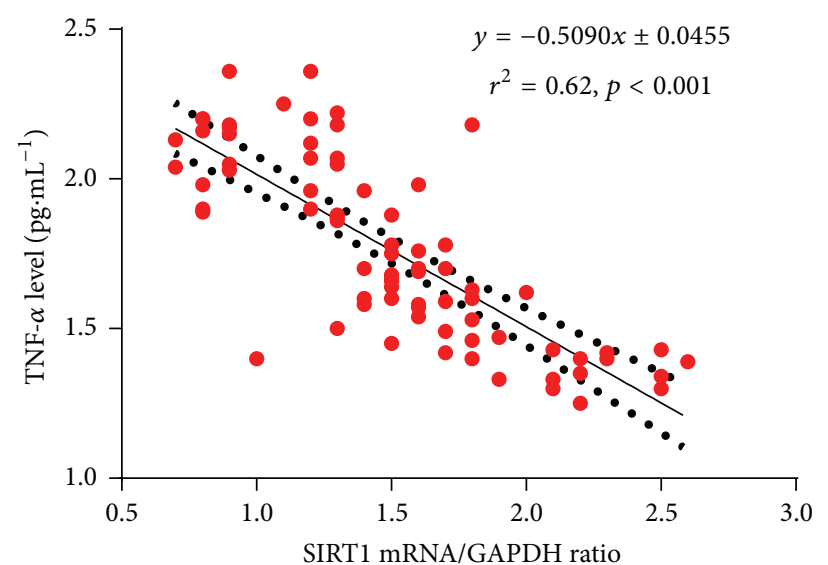

(b)

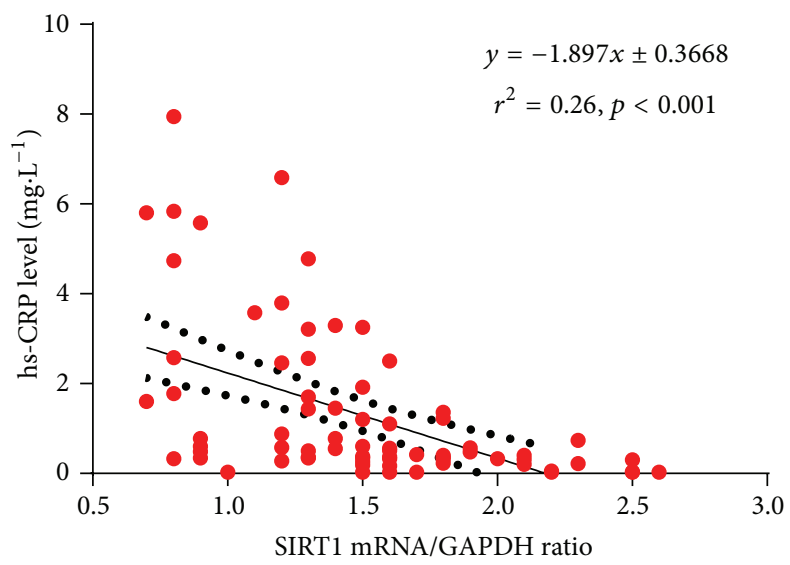

(d)

FIGURE 4: Correlation between levels of inflammatory cytokines and SIRT1 expression. SIRT1 expression was negatively correlated with plasma IL-6 (a), TNF- $\alpha$ (b), MCP-1 (c), and hs-CRP (d); $p<0.05$.

pancreas, and skeletal muscle [35]. Moreover, SIRT1 activation reduced diabetes symptoms and insulin secretion and delayed the onset of insulin resistance in diabetic rats [36]. On the other hand, Lagouge et al. [37] reported that the SIRT1 activator resveratrol enhanced insulin sensitivity in vitro and attenuated high fat diet-induced obesity and insulin resistance in vivo. This compound has also been shown to not only improve glucose metabolism and vascular function in patients with prediabetes but also improve left ventricle diastolic function and endothelial function in patients with CAD [38, 39]. Similarly, highly selective SIRT1-activating compounds improved insulin sensitivity, lowered plasma glucose, and increased mitochondrial capacity in diet-induced obese and genetically obese mice [40]. Finally, the SIRT1 activator SRT2104 was also shown to significantly affect serum cholesterol, LDL, and triglycerides [41].

However, these results remain controversial. Poulsen et al. [42] found that resveratrol did not affect blood pressure, resting energy expenditure, oxidation of lipid, ectopic or visceral fat, and inflammatory and metabolic biomarkers. Similarly, Semba et al. [43] reported that total urinary resveratrol metabolites in adults were not associated with inflammatory markers and cardiovascular disease or predictive of all-cause mortality. Thus, large-scale clinical studies may be necessary to resolve these contradictory results.

Although our sample size was relatively large, there were several limitations. While we did not observe a relationship between SIRT1 expression and the severity of coronary lesions in patients with both CAD and T2DM, the exact role of SIRT1 in atherosclerosis is more profound than most studies reported. Moreover, the contribution of SIRT1 to glucolipid metabolism and inflammatory signaling is not well characterized. Whether SIRT1 prevents atherosclerosis in diabetic patients remains to be seen.

\section{Conclusions}

In summary, the data demonstrate that SIRT1 expression in peripheral blood mononuclear cells is not associated with the severity of coronary lesions in patients with CAD and T2DM. However, SIRT1 mRNA expression is significantly correlated with IL-6, TNF- $\alpha$, MCP-1, and hs-CRP levels. Further research is needed to characterize the effects of SIRT1 on the development of atherosclerotic lesions. 


\section{Disclosure}

Yuanmin Li and Jing Ni should be co-first authors.

\section{Competing Interests}

The authors declare that they have no competing interests.

\section{Acknowledgments}

This work was supported by grants from the National Natural Science Foundation of China (no. 81070107).

\section{References}

[1] S. H. Ley, O. Hamdy, V. Mohan, and F. B. Hu, "Prevention and management of type 2 diabetes: dietary components and nutritional strategies," The Lancet, vol. 383, no. 9933, pp. 19992007, 2014.

[2] P. S. Baweja, P. B. Sandesara, and M. J. Ashraf, "Asymptomatic coronary artery disease in type II diabetes," Missouri Medicine, vol. 111, no. 1, pp. 73-79, 2014.

[3] M. G. Zeadin, C. I. Petlura, and G. H. Werstuck, "Molecular mechanisms linking diabetes to the accelerated development of atherosclerosis," Canadian Journal of Diabetes, vol. 37, no. 5, pp. 345-350, 2013.

[4] L. H. Young, F. J. T. Wackers, D. A. Chyun et al., "Cardiac outcomes after screening for asymptomatic coronary artery disease in patients with type 2 diabetes the DIAD study: a randomized controlled trial," The Journal of the American Medical Association, vol. 301, no. 15, pp. 1547-1555, 2009.

[5] E. J. Armstrong, J. Waltenberger, and J. H. Rogers, "Percutaneous coronary intervention in patients with diabetes: current concepts and future directions," Journal of Diabetes Science and Technology, vol. 8, no. 3, pp. 581-589, 2014.

[6] G. R. Myers and W. S. Weintraub, "Coronary artery disease: revascularization strategies for patients with CAD and diabetes," Nature Reviews Cardiology, vol. 7, no. 7, pp. 364-366, 2010.

[7] M. J. Zellweger, M. Maraun, H. H. Osterhues et al., "Progression to overt or silent cad in asymptomatic patients with diabetes mellitus at high coronary risk: main findings of the prospective multicenter BARDOT trial with a pilot randomized treatment substudy," JACC: Cardiovascular Imaging, vol. 7, no. 10, pp. 10011010, 2014.

[8] K. M. Hirahatake, J. L. Slavin, K. C. Maki, and S. H. Adams, "Associations between dairy foods, diabetes, and metabolic health: potential mechanisms and future directions," Metabolism: Clinical and Experimental, vol. 63, no. 5, pp. 618627, 2014.

[9] M. Quiñones, O. Al-Massadi, J. Fernø, and R. Nogueiras, "Cross-talk between SIRT1 and endocrine factors: effects on energy homeostasis," Molecular and Cellular Endocrinology, vol. 397, no. 1-2, pp. 42-50, 2014.

[10] X. Hou, S. Xu, K. A. Maitland-Toolan et al., "SIRT1 regulates hepatocyte lipid metabolism through activating AMP-activated protein kinase," Journal of Biological Chemistry, vol. 283, no. 29, pp. 20015-20026, 2008.

[11] M. Kitada and D. Koya, "SIRT1 in type 2 diabetes: mechanisms and therapeutic potential," Diabetes and Metabolism Journal, vol. 37, no. 5, pp. 315-325, 2013.
[12] S. Stein, C. Lohmann, N. Schäfer et al., "SIRT1 decreases Lox-1mediated foam cell formation in atherogenesis," European Heart Journal, vol. 31, no. 18, pp. 2301-2309, 2010.

[13] Y. Hu, L. Wang, S. Chen et al., "Association between the SIRT1 mRNA expression and acute coronary syndrome," Journal of Atherosclerosis and Thrombosis, vol. 22, no. 2, pp. 165-182, 2015.

[14] A. Breitenstein, C. A. Wyss, R. D. Spescha et al., "Peripheral blood monocyte sirtl expression is reduced in patients with coronary artery disease," PLoS ONE, vol. 8, no. 1, Article ID e53106, 2013.

[15] R. Guo, Y. Su, B. Liu, S. Li, S. Zhou, and Y. Xu, "Resveratrol suppresses oxidised low-density lipoprotein-induced macrophage apoptosis through inhibition of intracellular reactive oxygen species generation, LOX-1, and the p38 MAPK pathway," Cellular Physiology and Biochemistry, vol. 34, no. 2, pp. 603-616, 2014.

[16] S. Stein, N. Schäfer, A. Breitenstein et al., "SIRT1 reduces endothelial activation without affecting vascular function in ApoE-/mice," Aging, vol. 2, no. 6, pp. 353-360, 2010.

[17] S. Carter, S. Miard, C. Roy-Bellavance et al., "Sirtl inhibits resistin expression in aortic stenosis," PLoS ONE, vol. 7, no. 4, Article ID e35110, 2012.

[18] H.-T. Zeng, Y.-C. Fu, W. Yu et al., "SIRT1 prevents atherosclerosis via liver-X-receptor and NF- $\kappa \mathrm{B}$ signaling in a U937 cell model," Molecular Medicine Reports, vol. 8, no. 1, pp. 23-28, 2013.

[19] American Diabetes Association, "Classification and diagnosis of diabetes," Diabetes Care, vol. 38, supplement, pp. S8-S16, 2015.

[20] SYNTAX Score, http://www.syntaxscore.com.

[21] G. Sianos, M. A. Morel, A. P. Kappetein et al., "The SYNTAX Score: an angiographic tool grading the complexity of coronary artery disease," EuroIntervention, vol. 1, no. 2, pp. 219-227, 2005.

[22] L. R. Staimez, M. B. E. Weber, and E. W. Gregg, "The role of lifestyle change for prevention of cardiovascular disease in diabetes," Current atherosclerosis reports, vol. 16, no. 12, article 460, 2014.

[23] R. Guo, B. Liu, K. Wang, S. Zhou, W. Li, and Y. Xu, "Resveratrol ameliorates diabetic vascular inflammation and macrophage infiltration in $\mathrm{db} / \mathrm{db}$ mice by inhibiting the NF- $\kappa \mathrm{B}$ pathway," Diabetes and Vascular Disease Research, vol. 11, no. 2, pp. 92102, 2014.

[24] R. Guo, W. Liu, B. Liu, B. Zhang, W. Li, and Y. Xu, "SIRT1 suppresses cardiomyocyte apoptosis in diabetic cardiomyopathy: an insight into endoplasmic reticulum stress response mechanism," International Journal of Cardiology, vol. 191, pp. 36-45, 2015.

[25] M. Kitada, S. Kume, K. Kanasaki, A. Takeda-Watanabe, and D. Koya, "Sirtuins as possible drug targets in type 2 diabetes," Current Drug Targets, vol. 14, no. 6, pp. 622-636, 2013.

[26] A. B. Crujeiras, D. Parra, E. Goyenechea, and J. A. Martínez, "Sirtuin gene expression in human mononuclear cells is modulated by caloric restriction," European Journal of Clinical Investigation, vol. 38, no. 9, pp. 672-678, 2008.

[27] S. V. de Kreutzenberg, G. Ceolotto, I. Papparella et al., "Downregulation of the longevity-associated protein sirtuin 1 in insulin resistance and metabolic syndrome: potential biochemical mechanisms," Diabetes, vol. 59, no. 4, pp. 1006-1015, 2010.

[28] R. Song, W. Xu, Y. Chen, Z. Li, Y. Zeng, and Y. Fu, “The expression of sirtuins 1 and 4 in peripheral blood leukocytes from patients with type 2 diabetes," European Journal of Histochemistry, vol. 55, no. 1, article e10, 2011. 
[29] R. Caruso, I. Marafini, E. Franzè et al., "Defective expression of SIRT1 contributes to sustain inflammatory pathways in the gut," Mucosal Immunology, vol. 7, no. 6, pp. 1467-1479, 2014.

[30] G. Ceolotto, S. V. De Kreutzenberg, A. Cattelan et al., "Sirtuin 1 stabilization by HuR represses TNF- $\alpha$-and glucose-induced E-selectin release and endothelial cell adhesiveness in vitro: relevance to human metabolic syndrome," Clinical Science, vol. 127, no. 7, pp. 449-461, 2014.

[31] A. Storka, G. Führlinger, M. Seper et al., "E. coli endotoxin modulates the expression of sirtuin proteins in PBMC in humans," Mediators of Inflammation, vol. 2013, Article ID 876943, 6 pages, 2013.

[32] S. Matsushima and J. Sadoshima, "The role of sirtuins in cardiac disease," American Journal of Physiology - Heart and Circulatory Physiology, vol. 309, no. 9, pp. H1375-H1389, 2015.

[33] I. Gorenne, S. Kumar, K. Gray et al., "Vascular smooth muscle cell sirtuin 1 protects against dna damage and inhibits atherosclerosis," Circulation, vol. 127, no. 3, pp. 386-396, 2013.

[34] N. D’Onofrio, M. Vitiello, R. Casale, L. Servillo, A. Giovane, and M. L. Balestrieri, "Sirtuins in vascular diseases: emerging roles and therapeutic potential," Biochimica et Biophysica ActaMolecular Basis of Disease, vol. 1852, no. 7, pp. 1311-1322, 2015.

[35] S. Kume, T. Uzu, A. Kashiwagi, and D. Koya, "SIRT1, a calorie restriction mimetic, in a new therapeutic approach for type 2 diabetes mellitus and diabetic vascular complications," Endocrine, Metabolic and Immune Disorders-Drug Targets, vol. 10, no. 1, pp. 16-24, 2010.

[36] H.-C. Su, L.-M. Hung, and J.-K. Chen, "Resveratrol, a red wine antioxidant, possesses an insulin-like effect in streptozotocininduced diabetic rats," American Journal of PhysiologyEndocrinology and Metabolism, vol. 290, no. 6, pp. E1339-E1346, 2006.

[37] M. Lagouge, C. Argmann, Z. Gerhart-Hines et al., "Resveratrol improves mitochondrial function and protects against metabolic disease by activating SIRT1 and PGC-1 $\alpha$," Cell, vol. 127, no. 6, pp. 1109-1122, 2006.

[38] J. P. Crandall, V. Oram, G. Trandafirescu et al., "Pilot study of resveratrol in older adults with impaired glucose tolerance," Journals of Gerontology A: Biological Sciences and Medical Sciences, vol. 67, no. 12, pp. 1307-1312, 2012.

[39] K. Magyar, R. Halmosi, A. Palfi et al., "Cardioprotection by resveratrol: a human clinical trial in patients with stable coronary artery disease," Clinical Hemorheology and Microcirculation, vol. 50, no. 3, pp. 179-187, 2012.

[40] J. C. Milne, P. D. Lambert, S. Schenk et al., "Small molecule activators of SIRT1 as therapeutics for the treatment of type 2 diabetes," Nature, vol. 450, no. 7170, pp. 712-716, 2007.

[41] V. Libri, A. P. Brown, G. Gambarota et al., "A pilot randomized, placebo controlled, double blind phase I trial of the novel SIRT1 activator SRT2104 in elderly volunteers," PLOS ONE, vol. 7, no. 12, Article ID e51395, 2012.

[42] M. M. Poulsen, P. F. Vestergaard, B. F. Clasen et al., "Highdose resveratrol supplementation in obese men: an investigatorinitiated, randomized, placebo-controlled clinical trial of substrate metabolism, insulin sensitivity, and body composition," Diabetes, vol. 62, no. 4, pp. 1186-1195, 2013.

[43] R. D. Semba, L. Ferrucci, B. Bartali et al., "Resveratrol levels and all-cause mortality in older community-dwelling adults," JAMA Internal Medicine, vol. 174, no. 7, pp. 1077-1084, 2014. 


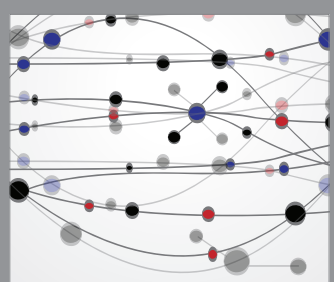

The Scientific World Journal
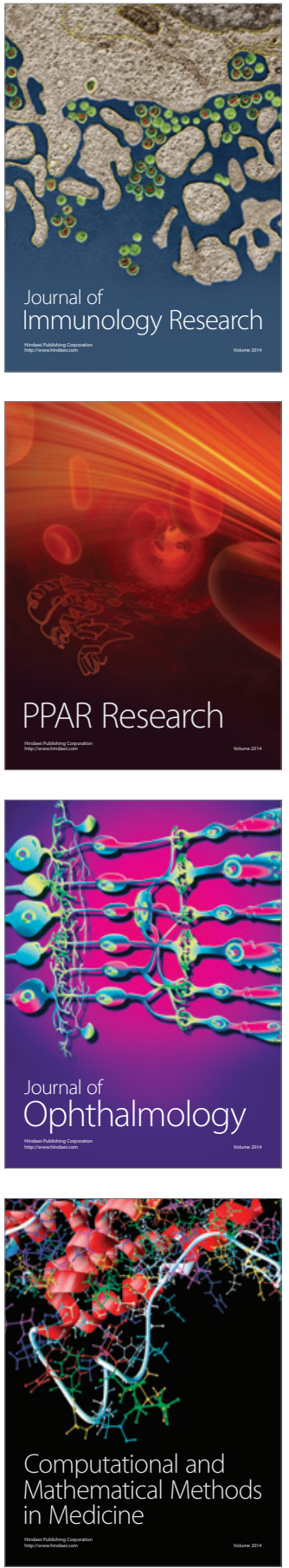

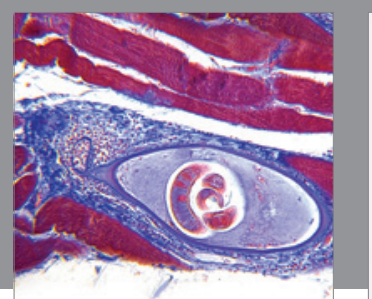

Gastroenterology Research and Practice

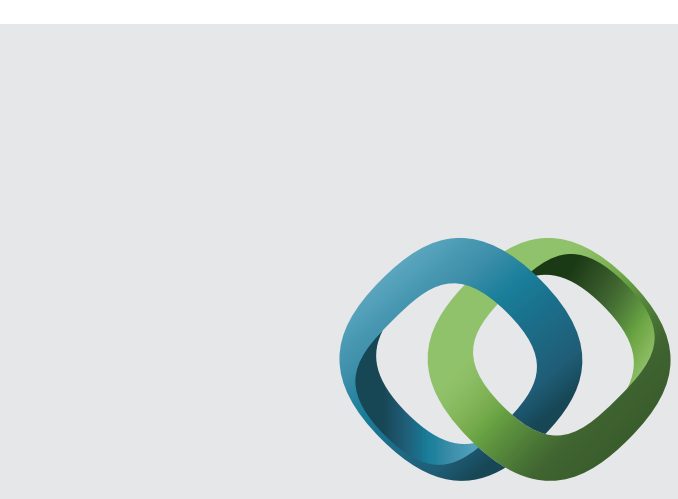

\section{Hindawi}

Submit your manuscripts at

http://www.hindawi.com
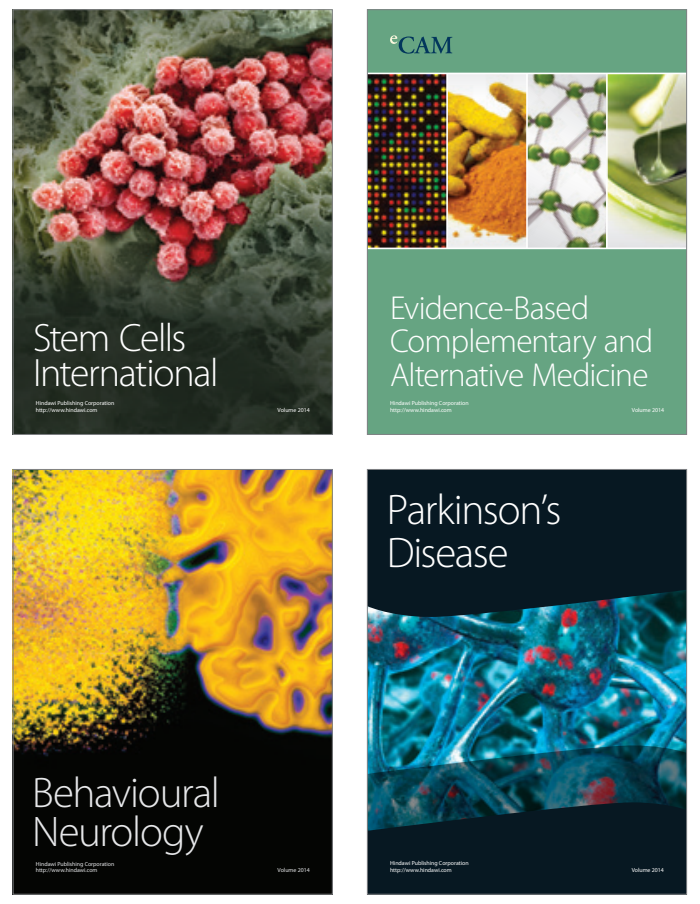
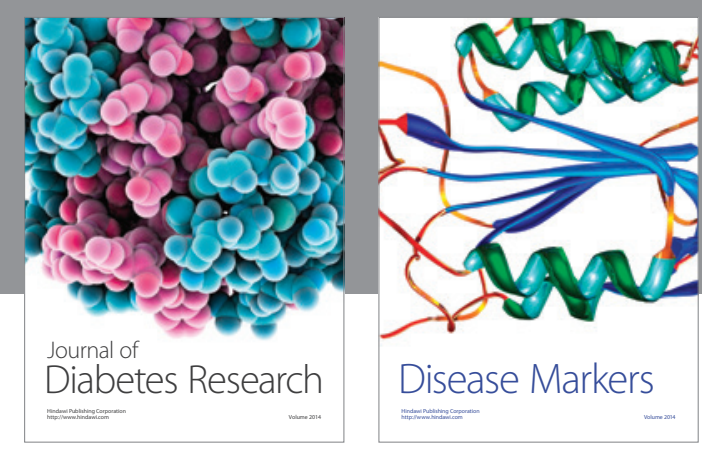

Disease Markers
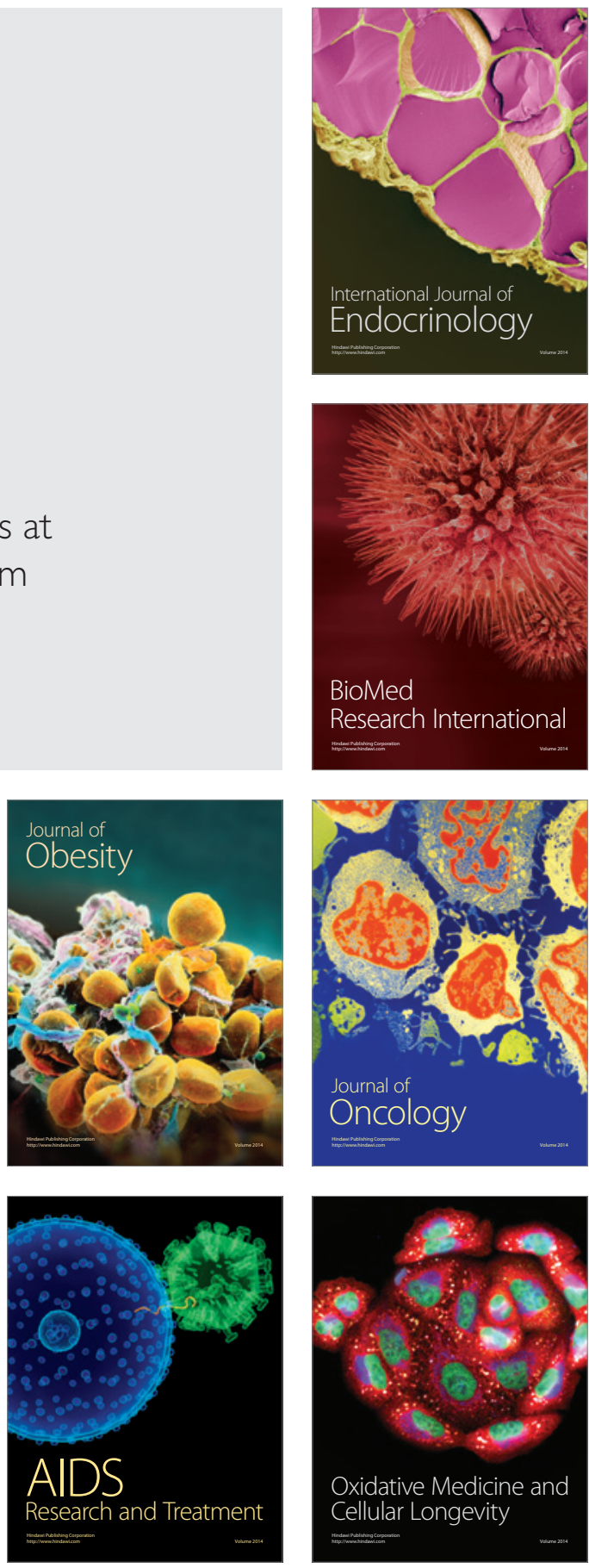